2. Kapitel

\title{
Gebrauch und Funktionieren im Bezug auf Reaktion
}

Wenn sich der Leser meine im letzten Kapitel geschilderten Erfahrungen vergegenwärtigt, wird er sich daran erinnern, wie mir an einem gewissen Punkt meiner Untersuchungen klar wurde, dass meine Reaktion auf einen ganz bestimmten Stimulus immer das Gegenteil von dem war, was ich eigentlich beabsichtigt hatte. Und als ich der Ursache nachging, erwiesen sich die Sinneseindrücke (Fühlen) beim Gebrauch meiner Mechanismen als derart unzuverlässig, dass ich mit einem Selbstgebrauch darauf reagierte, den ich zwar als richtig empfand, der sich aber in Wirklichkeit und für meine Zwecke allzu häufig als falsch erwies.

Ich weise auf diesen Punkt hin, weil bei ausnahmslos allen Schülern, die ich während meiner langjährigen Lehrtätigkeit in der Steuerung eines verbesserten Gebrauchs ihrer selbst unterrichtete, eine unterschiedlich starke Unzuverlässigkeit der Sinneswahrnehmung vorlag. Diese übte genau wie bei mir - einen schädlichen Einfluss auf ihren Gebrauch und auf ihr Funktionieren aus und folglich auch auf die Art, wie sie auf Stimuli reagierten. Deshalb bin ich überzeugt, dass die Unzuverlässigkeit der Sinneswahrnehmungen tatsächlich von grösster Bedeutung ist, wenn es um die Art geht, wie der Mensch seine Reaktionen kontrollieren kann.

Hinsichtlich der Kontrolle menschlicher Reaktionen ergibt sich noch ein anderer wichtiger Punkt: Dank meiner Entdeckung der primären Kontrolle konnte ich meine Sinneseinschätzung in Bezug auf den Gebrauch meiner Mechanismen verbessern, was auch zu einem besseren Funktionieren meines gesamten Organismus führte. Als sich durch die bewusste Anwendung der primären Kontrolle eine neue Art des Gebrauchs bei mir entwickelt hatte, konnte ich beim Eintreffen des Stimulus, die Stimme zu gebrauchen und zu rezitieren, meine instinktive Fehlsteuerung, 
die den alten, schädlichen Gebrauch von Kopf, Hals und Stimmorganen sowie meine Heiserkeit verursacht hatte, unterbinden und durch eine bewusste Steuerung ersetzen, die zu einem neuen Gebrauch von Kopf, Hals und Stimmorganen führte und keine Heiserkeit mehr nach sich zog.

Dies bedeutete, dass der Stimulus, meine Stimme zu gebrauchen, nicht länger die alte, abträgliche Reflexhandlung auslöste, mit der ich gewohnheitsmässig reagierte, indem ich den Kopf nach hinten und nach unten zog und so die Gestalt verkürzte, sondern es entwickelte sich eine neue Reflexhandlung, bei der ich den Kopf nach vorne und nach oben brachte, um die Gestalt zu verlängern, was sich in der Folge als die angemessenere Reaktion auf diesen Stimulus herausstellte.

Durch die Anwendung der primären Kontrolle konnte ich meine Reaktion auf den Stimulus zu sprechen so verbessern, dass das Rezitieren mir keine Heiserkeit mehr verursachte. Diese Tatsache nun beweist, dass ich gleich zu Anfang meiner Untersuchungen ein praktisches Mittel gefunden hatte, das meine gewohnheitsmässige Reflexhandlung als natürliche Folge des angewandten Verfahrens "neu konditionierte» und allmäblich die neue Reflextätigkeit herbeiführte, die ihrerseits wiederum mit neuen und besseren allgemeinen Zustandsbedingungen des Gebrauchs und des Funktionierens einherging. ${ }^{1}$

Die Resultate, die ich durch das beschriebene Verfahren (siehe S. 5 ff.) erzielte, zeigen tatsächlich, wie eine durch die Fehlsteuerung des Gebrauchs hervorgerufene schädliche Reflextätigkeit bewusst in Schach gehalten werden kann, und dies sogar angesichts einer gewissen Erregung, die sich bei dieser Vorgehensweise einstellt. ${ }^{2}$

Darüber hinaus hat mich diese Erfahrung gelehrt, dass die Resultate dieser «Neu- Konditionierung» ruhig sich selbst überlassen werden können, wenn erst das Wissen, wie die primäre Kontrolle zu steuern ist, zu einer

1 In diesem Zusammenhang dürfte folgender Auszug aus einer Vorlesung von Interesse sein, die Dr. A. Murdoch, Bexhill-on-Sea, am 6. März 1928 im St. Andrews (James Mackenzie) Institut hielt: «Herr Alexander hat die auf seiner praktischen Arbeit gründende Theorie aus Beobachtungen der Bewegungen des Körpers als Ganzem gewonnen und dabei ungewöhnlich scharfsinnig von den damit verbundenen, verlorengegangenen oder brachliegenden unwillkürlichen Reflexen Gebrauch gemacht. Und indem er diese als neue, bedingte Reflexe wiederherstellte, hat er den Grundstein gelegt für eine neue Betrachtungsweise von Krankheit, Diagnose und Behandlung.»

2 Bei dieser Vorgehensweise beginnt der Einzelne mit dem bewussten Erteilen von Direktiven für die Mittel, mit denen er ein bestimmtes Ziel erreichen will. Im kritischen Moment, wenn dieses Ziel verwirklicht werden soll, trifft er jedoch eine neue Entscheidung und beschliesst, mit diesen «Mitteln-wodurch» entweder das ursprüngliche oder ein anderes beliebiges Ziel zu erreichen. 
verbesserten Gebrauchsweise der Mechanismen im ganzen Organismus geführt hat. Professor John Dewey schreibt: «Die Wissenschaft ist schliesslich eine Sache des perfektionierten Könnens im Durchführen von Untersuchungen, ... kein fertiges oder in sich abgeschlossenes Etwas, sondern das Ergebnis einer bestimmten Technik.» 3

Es war mir also offensichtlich gelungen, durch eine veränderte Steuerung des Gebrauchs eine bewusste Kontrolle meiner Reaktionsweise zu bewirken. Der Leser wird daher verstehen, weshalb es, um einen veränderten Gebrauch herbeizuführen, von vorrangiger Bedeutung ist, eine instinktive durch eine bereusste Steuerung zu ersetzen. Und er wird auch begreifen, weshalb ich glaube, dass sich die Kenntnisse der Mittel, durch die eine solche Veränderung hervorgerufen werden kann, in allen Bereichen von Erziehungsarbeit als unschätzbar wertvoll erweisen dürften.

Als ich mich in der Praxis dann mit den Schwierigkeiten und Bedürfnissen meiner Schüler auseinandersetzen musste, haben sich meine Erfahrungen bei der Bewältigung meiner eigenen Probleme als ungemein nützlich erwiesen. Vor allem zeigten sie mir, dass ich meine Schüler nicht dazu bringen konnte, das Funktionieren ihrer Organe, Systeme oder Reflexe direkt zu kontrollieren. Indem ich sie jedoch in der bewussten Anwendung der primären Kontrolle des Gebrauchs unterwies, verschaffte ich ihnen Zugang $\mathrm{zu}$ den Mitteln, mit denen sie ihr allgemeines Funktionieren indirekt kontrollieren konnten. Wie die Erfahrung gezeigt hat, erwies sich dieses Prinzip, das ich in die Anwendung meiner Technik integriert habe, als vollkommen gerechtfertigt, und ich sehe auch bis heute keinen Grund, davon abzuweichen. Im Gegenteil, ich bin dank meiner zunehmenden Erfahrung immer mehr davon überzeugt, dass wir schwerlich jemals eine Methode entwickeln werden, mit der unserem Problem der Steuerung des bewussten - oder wie es manchmal heisst, des «bedingten» Verhaltens beizukommen wäre. Es sei denn, wir erarbeiteten eine bewusste Steuerung des Gebrauchs und machten die mit diesem Gebrauch verknüpfte und sich stets verfeinernde Einschätzung der Sinneswahrnehmungen zum vordringlichen Anliegen all jener, die sich beruflich in den unterschiedlichsten Bereichen mit dem Problem der menschlichen Reaktionsweise zu befassen haben.

Bei der Debatte über die Reaktionsweise des Menschen kann man von einigen wohlbekannten Tatsachen ausgehen:

Die menschliche Aktivität kann man zunächst hauptsächlich als einen Prozess unablässigen Reagierens auf Stimuli betrachten, die wir von innen 
oder von aussen empfangen. Der erste Atemzug eines Neugeborenen ist die Reaktion auf einen Stimulus des Atmungszentrums, und das Kind bleibt nur so lange ein lebender Organismus, wie es fähig ist, Stimuli zu empfangen und darauf zu reagieren. Der Mensch kann Stimuli lediglich über die Sinnesmechanismen empfangen; angenommen, man könnte diese Sinnesmechanismen daran hindern, einen Stimulus zu empfangen, dann wäre keine Reaktion und daher auch keine weitere Aktivität möglich, ein Zustand, der dem Leben als solchem ein Ende bereiten würde.

Jegliche Aktivität ist die Reaktion auf einen durch die Sinnesmechanismen empfangenen Stimulus, und ist dies einmal erkannt, kann man keine Aktivität mehr als gänzlich "psychisch» oder als gänzlich "physisch» bezeichnen. Man könnte höchstens sagen, dass bei den einen Aktivitäten die "psychische» und bei den anderen die "physische» Komponente überwiegt. Nehmen wir beispielsweise das Heben eines Arms, einen Akt also, den viele kurzerhand "physisch» nennen würden. Wenn wir allerdings berücksichtigen, was zwischen dem Eintreffen dieses spezifischen Stimulus und dem Ausführen dieses Aktes geschieht, dann wird uns klar, dass es sich hier um ein Zusammenwirken handelt, das nicht nur sogenannte "physische» Abläufe beinhaltet, sondern auch solche, die gemeinhin als «mental» betrachtet werden. Wenn uns nun der Stimulus zum Heben eines Arms erreicht, stellen wir uns dabei bekanntlich einen «mentalen» Akt vor, und auf diese Vorstellung folgt ein weiterer sogenannter «mentaler» Vorgang, nämlich der des Einwilligens oder des Verweigerns, auf diesen Stimulus zu reagieren. Bei Verweigern unterbinden wir die Reaktion, die das Heben des Armes auslösen würde: der Arm wird nicht gehoben. Bei Einwilligung tritt die Steuerung der für das Heben des Armes notwendigen Mechanismen in Kraft und es werden Botschaften übermittelt, die eine Kontraktion bestimmter und eine Lockerung anderer Muskelgruppen bewirken: der Arm wird gehoben.

Man muss in diesem Zusammenhang jedoch unbedingt darauf hinweisen, dass die Steuerung des Selbstgebrauchs bei den meisten Menschen gewohnheitsmässig und instinktiv erfolgt: Ist die Einwilligung erteilt, auf einen Stimulus zu reagieren und einen bestimmten Akt auszuführen, wird dieser Akt «instinktiv» - wie wir sagen - ausgeführt, das heisst wir haben keine bewusste Vorstellung darüber, welcher Gebrauch steuernder Mechanismen notwendig wäre, um ihn zufriedenstellend auszuführen.

Angesichts der vorherrschenden und zunehmenden Unzuverlässigkeit unserer Sinneseinschätzung führt diese instinktive Steuerung - wie mein eigenes Beispiel klar gezeigt hat - mit der Zeit leider zu einer immer ausgeprägteren Form von Fehlsteuerung, die sich schädlich auf das Funktionieren und die daraus resultieren Reaktionen auswirkt. ${ }^{4}$ 
Unbefriedigende Reaktionen dieser Art manifestieren sich in Form von Beeinträchtigungen, in sogenanntem "psychischem» oder "physischem» Versagen, und treten als Anzeichen von Störungen oder Krankheiten auf, die normalerweise darauf hindeuten, dass auch im gesamten Organismus ein gewohnheitsmässig falscher Gebrauch und falsches Funktionieren vorliegt. ${ }^{5}$ Nach meinen Erfahrung mit solchen Fällen verschwinden diese "Symptome» allmählich und dürften von Gesundheit und Wohlergehen oder von angemessenen Reaktionen abgelöst werden, wenn man einen neuen und zufriedenstellenden Steuerungsgebrauch der Mechanismen und damit eine Verbesserung der damit verbundenen Funktionsweise herbeiführen kann. Ich wage deshalb zu behaupten, dass bei allen spezifischen Symptomen das Vermeiden einer Fehlsteuerung ein vordringliches Anliegen ist, da diese zu einer falschen Gebrauchs- und Funktionsweise führt. An ihrer Stelle muss eine neue und zufriedenstellende Steuerung aufgebaut werden, die eine verbesserte Gebrauchs- und Funktionsweise im ganzen Organismus hervorruft.

Das indirekte Vorgehen stimmt vollkommen mit dem Prinzip überein, dass der menschliche Organismus ein unteilbares Ganzes ist. Und dieses Prinzip der Unteilbarkeit arbeitet zu unserem Vorteil, wenn die Mittel erkannt sind, mit denen der Gebrauch der Mechanismen in einem aktiven Zusammenwirken so gesteuert werden kann, wie ich es darzulegen versuche. Allerdings gibt es auch eine Kehrseite der Medaille: Es liegt im Wesen der Ganzheit, dass jede Veränderung in einem Teil auch eine Veränderung im Ganzen nach sich zieht. Wenn also die eng miteinander verbundenen Teile des menschlichen Organismus zu einem Ganzen verknüpft sind, führt jeder Versuch, eine wesentliche Funktionsänderung eines Teils herbeizuführen, unweigerlich zu Veränderungen des Gebrauchs und des Zusammenspiels im ganzen Organismus. Ist das Zusammenspiel im Gebrauch der Mechanismen jedoch im ganzen Organismus fehlerhaft, bringt jeder Versuch, eine einzelne Unzulänglichkeit auszumerzen, unweigerlich eine andere Stelle aus dem Gleichgewicht, wenn er nicht mit

4 Dies ist eine demonstrierbare und beweisbare Tatsache, die im Verlaufe meiner Untersuchungen zutage getreten ist. (Siehe Kapitel 1.)

5 Hier möchte ich folgendes klarstellen: Immer wenn ich im Zusammenhang mit dem menschlichen Organismus von "Gebrauch und Funktionieren" spreche, weise ich nicht lediglich auf die mechanische Aktivität als solche hin, sondern schliesse immer auch sämtliche Manifestationen menschlicher Aktivität mit ein, wie etwa Auffassen oder Verstehen, Zustimmung geben oder Zustimmung verweigern, denken, überlegen, Befehle erteilen usw., denn die Manifestation solcher Aktivitäten kann nicht abgespalten werden vom Gebrauch der Mechanismen und dem damit verbundenen Funktionieren des Organismus. 
Veränderungen und Verbesserungen dieses gesamten Zusammenspiels einhergeht. ${ }^{6}$ Diese Gefahr jedoch wird von denen, die Krankheiten oder Behinderungen diagnostizieren und behandeln, selten erkannt. Ich aber kann beweisen, dass im Vorgang des «Heilens» eines unerwünschten Symptoms andere Teile des Organismus in Mitleidenschaft gezogen werden und dort andersartige, weniger leicht erkennbare, aber oft schlimmere Defekte verursachen, obwohl die spezifische Behandlung nach aussen hin sogar erfolgreich scheinen mag. Dies ist die alte Geschichte von den sieben Teufeln. ${ }^{7}$

Wie mir die Ergebnisse meiner Unterrichtstätigkeit gezeigt haben, kann keine Diagnose vollständig sein, wenn ihr nicht jenes Prinzip des einheitlichen Zusammenspiels der Mechanismen des Organismus zugrunde liegt, das einen engen Zusammenhang zwischen der Art des Gebrauchs der Mechanismen und der Funktionstüchtigkeit des gesamten Organismus mit einbezieht.

In den folgenden Kapiteln werde ich nun mehrere Beispiele dafür anführen, wie Fachleute unterschiedlichster Bereiche dieses Prinzip missachten, wenn sie in ihrer Praxis bei einem Menschen eine Unzulänglichkeit oder eine Behinderung korrigieren müssen. Es kommt dadurch zu einer unvollständigen Diagnose, die den Behandlungsspielraum aller Fachrichtungen wesentlich einschränkt.

Ein Verfahren kann erst dann richtig beurteilt werden, wenn das ihm zugrunde liegende Prinzip überprüft wurde. Ist das Prinzip fragwürdig, muss das Verfahren, langfristig gesehen, scheitern. Deshalb möchte ich Sie bitten, die praktischen Verfahren, die ich nun unterbreite, nach dem Prinzip zu bewerten, das ihnen zugrunde liegt.

6 Siehe Kapitel 4, Seiten $51 \mathrm{ff}$.

7 Es ist interessant zu lesen, was Sir E. Holderness, eine anerkannte Kapazität auf dem Gebiet des Golfspiels, in diesem Zusammenhang im Evening Standard vom 17. März 1928 geschrieben hat: «Dies hier ist die wahre Geschichte eines Freundes, der sich grämte, weil sein Ball regelmässig einen Seitwärtsbogen nach rechts beschrieb. Voller Verzweiflung wandte er sich an einen Golflehrer, der ihm eine Schnellkur anbot, indem er ihn instruierte, den Schläger beim Abschlag so zu halten, dass die rechte Hand am oberen Ende und die linke darunter auflag. Dann forderte er ihn auf, einfach mit Selbstvertrauen abzuschlagen. Es ist eine wunderbare Geschichte, denn der Seitwärtsbogen nach rechts verschwand, und für einen Nachmittag lang spielte mein Freund göttlich. Aber wo ein Teufel gesteckt hatte, ersetzten ihn nun sieben andere, denn anschliessend musste sich mein Freund wochen- und monatelang mit Bällen abquälen, die einen Seitwärtsbogen nach links beschrieben oder die, weil er sie zu weit unten abschlug, eine viel zu kurze Distanz zurücklegten. Schliesslich war mein Freund in einer schlimmeren Lage als zuvor.» 